Proc. IEEE Intl. Conf. Image Proc., Sept. 1996, vol. I, pp. 81-84

\title{
RESTORATION OF UNCERTAIN BLURS USING AN ERROR IN VARIABLES CRITERION
}

\author{
G. Sharma and H. J. Trussell \\ Electrical \& Computer Engineering Dept. \\ North Carolina State University, Raleigh, NC 27695-7911
}

\begin{abstract}
In image-restoration problems involving a known blur, set-theoretic estimation provides an effective framework for incorporation of both noise statistics and a priori information. However, the sets formulated for use with known blurs are not suitable for situations involving unknown stochastic blurs. In this paper, new sets based on an error in variables criterion are developed, which are more appropriate for restoration of uncertain blurs. The conditions for convexity of these sets are rigorously established. Through 1-D simulations, the settheoretic restoration scheme utilizing these sets is compared with the stochastic MMSE filter and with settheoretic schemes for stochastic blurs developed earlier.
\end{abstract}

\section{INTRODUCTION}

In several imaging applications, such as ground-based astronomy and X-ray photography, the image recording process involves an uncertain blur $[1,2]$. In restoring such images, often the average blur is used in restoration schemes designed for images with known blur. Significant gains can be achieved if additional knowledge of the blur statistics is used in the restoration process. The stochastic minimum mean square (MMSE) filter [2]; the method of total least squares (TLS)[3], or error in variables regression; and the constrained total least squares (CTLS) technique [4, 5] are all motivated by this idea. While these estimation schemes are statistically sound, they do not permit use of other a priori knowledge of the signal in the estimation procedure.

Set theoretic estimation [6] provides a flexible framework for incorporation of a priori knowledge into estimates of the signal. If available knowledge about the signal can be represented in terms of sets $\left\{S_{i}\right\}_{i=1}^{m}$, in which the signal must lie, a point in the intersection of all these constraint sets, i.e., in

$$
S_{0}=\bigcap_{i=1}^{m} S_{i},
$$

is used as an estimate for the signal.

Obviously, set theoretic estimation is useful only for problems for which there exists a procedure for computing this estimate. If all the sets, $\left\{S_{i}\right\}_{i=1}^{m}$, are closed and convex, the method of successive projections onto convex sets (POCS) is guaranteed to converge to a point in $S_{0}$ starting from any arbitrary initial estimate $[7,8]$. This result has been successfully exploited for a number of signal restoration problems with a known blur, and several convex sets have been defined based on prior knowledge and noise statistics for that case $[9,10]$.

In [11] the sets based on noise properties were modified to take into account the case of a stochastic blur. The modifications in [11] were primarily enlargements of the sets based on noise statistics, to incorporate the additional uncertainties introduced by inaccurate knowledge of the blur. In this paper, new sets describing the properties of the noise and the blur perturbations are defined in spatial and frequency domains. The convexity of the new sets is established by means of an elegant alternate characterization. Through onedimensional simulations, the performance of restoration based on the modified sets is compared with the stochastic MMSE filter and the approach in [11].

\section{MEASUREMENT MODEL}

For blurs arising from linear systems, the recorded data can be represented as

$$
\mathbf{g}_{N \times 1}=\mathbf{H}_{N \times N} \mathbf{f}_{N \times 1}+\boldsymbol{v}_{N \times 1}
$$

where the matrix $\mathbf{H}$ is the linear blur, $\mathbf{f}$ is the original signal to be restored, $\boldsymbol{v}$ is the measurement noise, and the subscripts indicate the dimensions of the vectors and matrices. For the case of stochastic blurs, $\mathbf{H}$ may be modeled as $\mathbf{H}=\overline{\mathbf{H}}+\boldsymbol{\Delta} \mathbf{H}$, where $\overline{\mathbf{H}}$ and $\boldsymbol{\Delta} \mathbf{H}$ represent the known and unknown parts of $\mathbf{H}$, respectively. It will be assumed here that the entries in $\boldsymbol{v}$ are samples of a zero mean white noise process with a variance of $\sigma_{v}^{2}$. 


\section{SETS BASED ON NOISE STATISTICS}

If the blur, $\mathbf{H}$, is known then under the hypothesis that the true signal is $\mathbf{y}$, the residual, $\mathbf{g}-\mathbf{H y}$, corresponds to the noise. In the method of least squares, the restoration is chosen as the image which minimizes the norm of this residual, whereas in set theoretic estimation [10] an upper bound is placed on the norm of the residual in agreement with noise statistics, to define the set [10]

$$
S_{v}=\left\{\mathbf{x} \mid\|\mathbf{g}-\mathbf{H} \mathbf{x}\|^{2} \leq \delta_{v}\right\}
$$

where $\delta_{v}$ is equal to $N \sigma_{v}^{2}$.

For the case when the blur is uncertain, the leastsquares paradigm is usually replaced by a (weighted) total least squares scheme[3] in which the total squared residual, $\tau\|\mathbf{E}\|_{F}^{2}+\|\boldsymbol{\eta}\|^{2}$, is minimized, where $\mathbf{E}$ and $\boldsymbol{\eta}$ are constrained to satisfy $(\overline{\mathbf{H}}+\mathbf{E}) \mathbf{x}=\mathbf{g}+$ $\boldsymbol{\eta},\|\cdot\|_{F}$ denotes the Frobenius norm [3], and $\tau$ is a positive weighting factor. The stochastic blur case was considered for set theoretic estimation in [11], where a modified noise process, $\boldsymbol{v}^{\prime}=\mathbf{g}-\overline{\mathbf{H}} \mathbf{f}=\boldsymbol{v}+\boldsymbol{\Delta} \mathbf{H f}$, was defined. This led to an enlarged set for the sample variance of the residual,

$$
S_{v}^{\prime}=\left\{\mathbf{x} \mid\|\mathbf{g}-\overline{\mathbf{H}} \mathbf{x}\|^{2} \leq \delta_{v}^{\prime}\right\}
$$

where $\delta_{v}^{\prime}=\delta_{v}+E\|\boldsymbol{\Delta} \mathbf{H f}\|^{2}$ is the variance of $\boldsymbol{v}^{\prime}$, with $E$ representing the expectation operator. For the purposes of restoration, the worst case value of $E \|$ $\mathbf{\Delta H f} \|^{2}$ given by $E\|\boldsymbol{\Delta} \mathbf{H}\|^{2}\|\mathbf{f}\|^{2}$ was used.

The total least squares approach can be used to motivate an alternative set that accounts for the unknown component of $\mathbf{H}$. First, note that the set $S_{v}$ may be rewritten as $S_{v}=\left\{\mathbf{x} \mid \exists \boldsymbol{\eta} \ni \mathbf{H x}=\mathbf{g}+\boldsymbol{\eta},\|\boldsymbol{\eta}\|^{2} \leq \delta_{v}\right\}$. In a similar fashion, based on error in variables regression, a set can be defined to account for both the noise and the unknown part of $\mathbf{H}$ as

$$
\begin{aligned}
S_{T L S}= & \{\mathbf{x} \mid \exists\{\mathbf{E}, \boldsymbol{\eta}\} \ni(\overline{\mathbf{H}}+\mathbf{E}) \mathbf{x}=\mathbf{g}+\boldsymbol{\eta}, \\
& \left.\tau\|\mathbf{E}\|_{F}^{2}+\|\boldsymbol{\eta}\|^{2} \leq \nu\right\},
\end{aligned}
$$

where $\tau$ and $\nu$ are positive parameters determined by the statistics of $\boldsymbol{\Delta} \mathbf{H}$ and $\boldsymbol{v}$.

In order to use the set, $S_{T L S}$, in POCS based signal restoration, it is necessary to establish its convexity and to determine the projection onto it. The implicit definition in (4) involving $\mathbf{E}, \boldsymbol{\eta}$ makes both these tasks rather difficult. The following theorem provides us an alternate characterization of $S_{T L S}$ simplifying both tasks. The conditions for convexity follow from a straightforward corollary. Both the theorem and the corollary are proved in [12].

Theorem $1 S_{T L S}=\left\{\mathbf{x} \in \mathbb{R}^{N} \mid\|\overline{\mathbf{H}} \mathbf{x}-\mathbf{g}\|^{2}-\frac{\nu}{\tau} \|\right.$ $\left.\mathbf{x} \|^{2}-\nu \leq 0\right\}$.
Corollary $1 S_{T L S}$ is closed and convex if $\sqrt{\frac{\nu}{\tau}}$ is less than or equal to the smallest singular value of $\mathbf{H}$.

\section{POWER SPECTRAL BOUNDS SETS}

If the blur is assumed to be shift invariant, the operators $\overline{\mathbf{H}}$ and $\boldsymbol{\Delta} \mathbf{H}$ are Toeplitz matrices determined by the known part, $\mathbf{h}$, and the unknown part, $\Delta \mathbf{h}$, of the stochastic impulse response, respectively. Under this assumption, for the known blur case, $N$ convex sets were defined in [10] by placing appropriate confidence limits on the periodogram of the residual. In [11] the sets were modified to account for stochastic impulse responses by expanding the bounds and making them frequency dependent, to get the sets

$S_{p}^{(k)}=\left\{\mathbf{x}|| G(k)-\left.\bar{H}(k) X(k)\right|^{2} \leq \delta_{p}^{\prime}(k)\right\}, 0<k<\frac{N}{2}$

where upper case letters represent the discrete Fourier transform (DFT) of their lower case counterparts,

$$
\delta_{p}^{\prime}(k)=\frac{\alpha N}{2} \sigma_{v}^{2}+\frac{\alpha N}{2} P_{f}(k) E|\Delta H(k)|^{2},
$$

$P_{f}(k)$ is the periodogram of $\mathbf{f}$, and $\alpha$ is a confidence factor determined for a given confidence level for a normalized chi-square random variable with two degrees of freedom.

A modification similar to that for the residual variance set can be used for the power spectral bounds (PSB) set, by defining the following sets,

$$
\begin{aligned}
S_{t}(k)= & \{\mathbf{x} \mid \exists \Delta, E \in \mathbb{C} \ni[\bar{H}(k)+\Delta] X(k)=G(k) \\
& \left.+E, \tau_{k}|\Delta|^{2}+|E|^{2} \leq \nu_{k}\right\}, 0<k<\frac{N}{2} .
\end{aligned}
$$

It can be shown that [12]

$$
\begin{aligned}
S_{t}(k)= & \left\{\mathbf{x}|| \bar{H}(k) X(k)-\left.G(k)\right|^{2}-\frac{\nu_{k}}{\tau_{k}}|X(k)|^{2}\right. \\
& \left.-\nu_{k}^{2} \leq 0\right\},
\end{aligned}
$$

from which it follows that $S_{t}(k)$ is a closed convex set if $|\bar{H}(k)|^{2} \geq \frac{\nu_{k}}{\tau_{k}}$.

Projections onto $S_{T L S}$ and $S_{t}(k)$ can readily be determined from their characterizations given above using standard non-linear programming techniques [13]. These are summarized in [12].

\section{EXPERIMENTAL RESULTS}

In order to test the effectiveness of the modified sets in restoration problems, the $1-\mathrm{D}$ restoration example 
from [11] was simulated. A stochastic impulse response with Gaussian mean given by,

$$
\bar{h}(n) \approx \frac{1}{8 \sqrt{2 \pi}} \exp \left(-\frac{n^{2}}{128}\right)
$$

was created by generating $M$ points between -128 and 127 , with the location of each point being a zero-mean Gaussian-distributed r.v. with a standard deviation of 8. The stochastic impulse response was then computed as

$$
h(n)=\frac{\text { number of points in }[n-0.5, n+0.5)}{M} .
$$

The mean of the stochastic impulse response, $\bar{h}(n)$, was assumed to be the known part of the impulse response. $E\left\{|\Delta H(k)|^{2}\right\}$ was also assumed to be known and computed by averaging over 1000 realizations of the stochastic impulse response. The noise was generated using a Gaussian random number generator, and the variance $\sigma_{v}^{2}$ was computed from the signal-to-noise ratio (SNR), defined as $10 \log _{10}\left(\|f\|^{2} / \sigma_{v}^{2}\right)$. The SNR was set at $30 \mathrm{~dB}$ for the simulations.

For comparison, restoration was performed using three different methods: the stochastic MMSE filter, the dynamic POCS (DPOCS) technique from [11], and POCS using the modified sets. The parameter $M$ which governs the uncertainty of the impulse response was set equal to 100 as in [11]. The stochastic MMSE filter is given by

$$
Q(k)=\frac{P_{f}(k) \bar{H}^{*}(k)}{P_{f}(k)\left(|\bar{H}(k)|^{2}+E|\Delta H(k)|^{2}\right)+\sigma_{v}^{2}} .
$$

The degraded signal was used as the initial estimate for both the POCS schemes.

The original signal and the degraded signal are shown in Fig. 1. The three estimates are shown in figures 2, 3 , and 4 for the stochastic MMSE, DPOCS, EVPOCS schemes, respectively. All plots include the actual signal for comparison. From the figures, it can be seen that the stochastic MMSE filter yields a smooth restored signal which does not fully capture the levels in the original signal and has negative lobes. For the DPOCS technique, the sets are rather conservative and hence the estimate is not significantly different from the degraded signal used as the initial estimate. The EVPOCS approach using the sets $S_{t}^{(k)}$ performs significantly better giving good resolution of the two signal peaks around $n=200$ and also some discrimination between the multiple signal levels in the central region.

\section{REFERENCES}

[1] H. Andrews and B. R. Hunt, Digital Image Restoration, Prentice Hall, Englewood Cliffs, NJ, 1977.

[2] L. Guan and R. K. Ward, "Deblurring random time-varying blur," J. Opt. Soc. Am. A, vol. 6, no. 11, pp. 1727-1737, November 1989.

[3] Gene H. Golub and Charles F. Van Loan, Matrix Computations, The Johns Hopkins University Press, Baltimore, MD, second edition, 1989.

[4] B. R. Hunt, "The application of constrained leastsquares to image restoration by digital computer," IEEE Trans. Computers, vol. 22, no. 9, pp. 805812, September 1973.

[5] V. Z. Mesarović, N. P. Galatsanos, and A. K. Katsaggelos, "Regularized constrained total least squares image restoration," IEEE Trans. on Image Proc., vol. 4, no. 8, pp. 1096-1107, August 1995.

[6] P. L. Combettes, "The foundations of set theoretic estimation," Proceedings of the IEEE, vol. 81, no. 2, pp. 182-208, February 1993.

[7] L. M. Bregman, "The method of successive projection for finding a common point of convex sets," Dokl. Akad. Nauk. USSR, vol. 162, no. 3, pp. 487490, 1965.

[8] L. G. Gubin, B. T. Polyak, and E. T. Raik, "The method of projections for finding the common point of convex sets," USSR Comput. Math. and Phys., vol. 7, no. 6, pp. 1-24, 1967.

[9] P. L. Combettes and H. J. Trussell, "The use of noise properties in set theoretic estimation," IEEE Trans. Sig. Proc., vol. 39, no. 7, pp. 1630-1641, July 1991.

[10] H. J. Trussell and M. R. Civanlar, "Feasible solution in signal restoration," IEEE Trans. ASSP, vol. ASSP-32, no. 4, pp. 201-212, April 1984.

[11] P. L. Combettes and H. J. Trussell, "Methods for restoration of signals degraded by a stochastic impulse response," IEEE Trans. ASSP, vol. 37, no. 3, pp. 393-401, March 1989.

[12] G. Sharma and H. J. Trussell, "Set theoretic signal restoration using an error in variables criterion," submitted to IEEE Trans. Image Proc.

[13] David G. Luenberger, Linear and Nonlinear Programming, Addison Wesley, Reading, MA, second edition, 1989. 


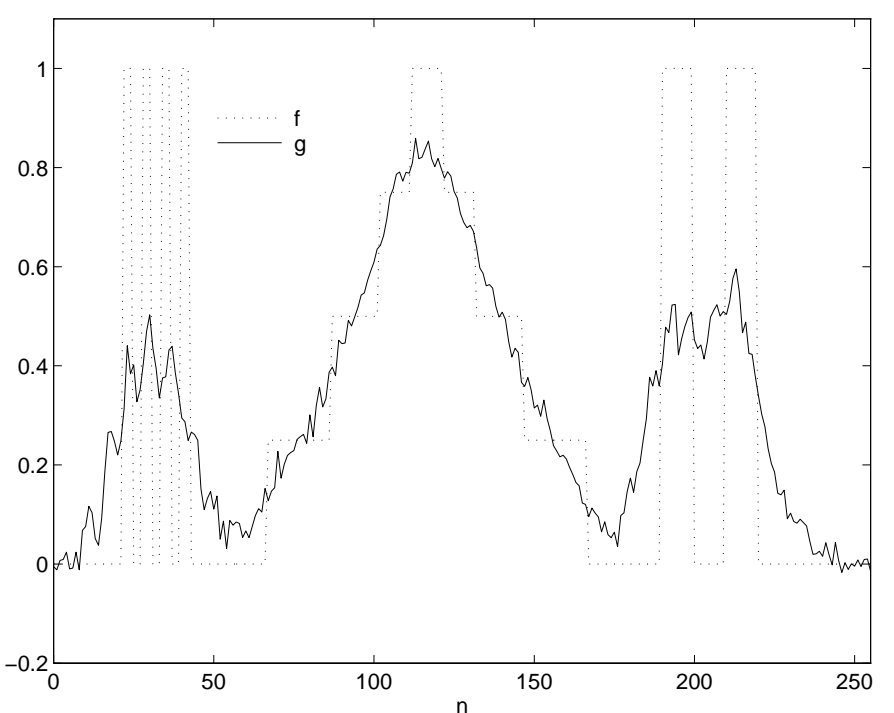

Figure 1: Original and degraded signals.

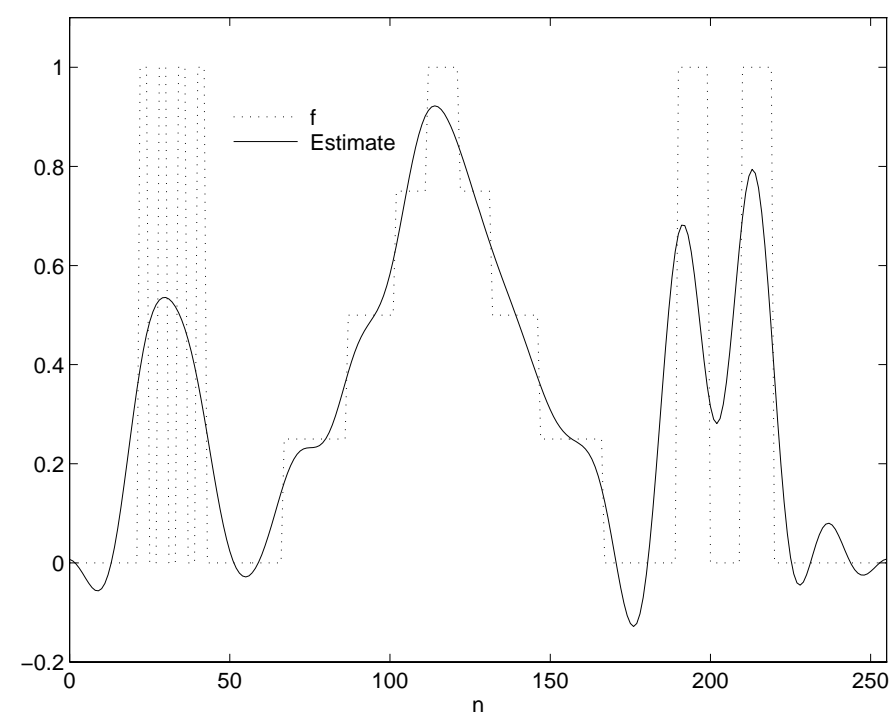

Figure 2: Stochastic MMSE Restoration.

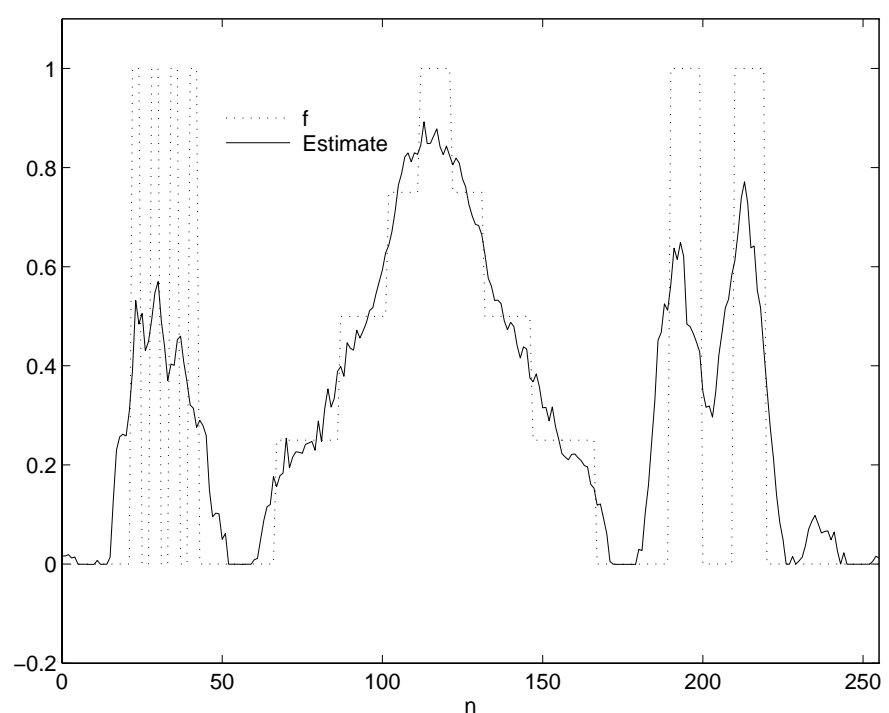

Figure 3: Dynamic POCS Restoration.

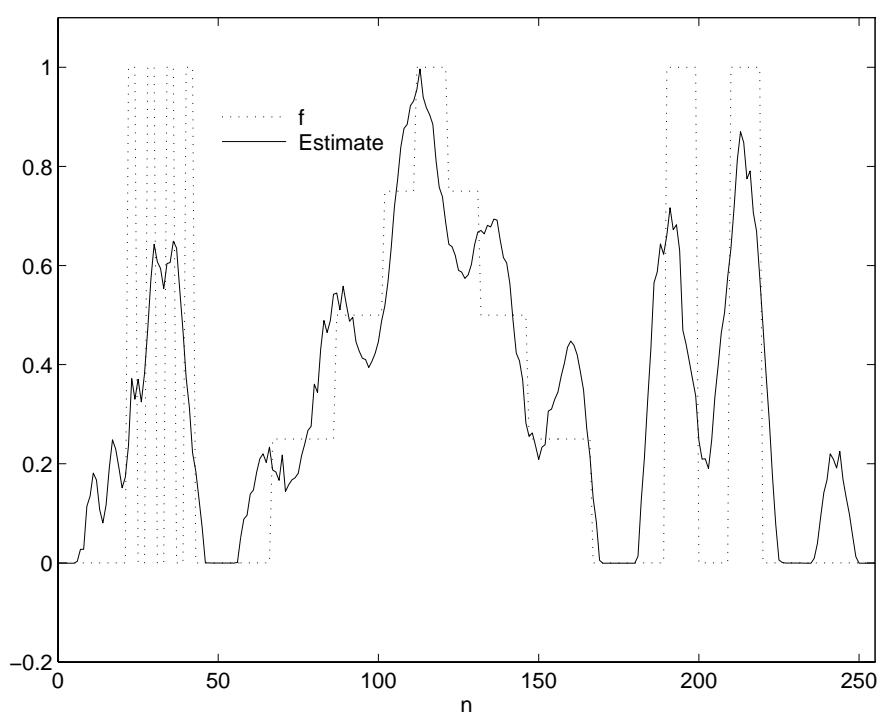

Figure 4: Error in Variables POCS Estimate. 